\title{
Fracturas desplazadas de epicóndilo medial. Revisión bibliográfica sistematizada
}

\author{
Dres. Rodrigo Matías Fratelli Álvarez, Viviana Teske†, Alejandro Cuneo \\ Clínica de Traumatología y Ortopedia Pediátrica, Facultad de Medicina, Universidad de la República
}

\section{Resumen}

Introducción: la fractura de epicóndilo medial representa 12\% a 20\% de las fracturas del codo pediátrico. Existe controversia en la indicación del tratamiento para las fracturas con un desplazamiento mayor de $2 \mathrm{~mm}$. El objetivo de este trabajo es revisar la indicación y los resultados de los diferentes tratamientos realizados en las fracturas de epicóndilo medial desplazadas más allá de $2 \mathrm{~mm}$.

Material y método: se utilizaron las bases de datos electrónicas PubMed y Bireme. La búsqueda arrojó un total de 313 artículos de los cuales nueve cumplían con los criterios de inclusión propuestos al iniciar la búsqueda.

Discusión: se analizaron y compararon los métodos de diagnóstico por imagen utilizados y su rendimiento, los diferentes tipos de tratamiento y las complicaciones.

Conclusión: no debe ser el desplazamiento de la fractura lo que guíe el tratamiento sino la energía traumática y la estabilidad en valgo del codo. Se han reportado excelentes resultados tanto con tratamiento conservador como quirúrgico de las fracturas desplazadas de epicóndilo medial. Faltan estudios comparativos con seguimiento a largo plazo de buen nivel de evidencia para recomendar uno u otro tratamiento en las fracturas desplazadas de epicóndilo medial.

Palabras clave: FRACTURAS DEL HÚMERO

ARTICULACIÓN DEL CODO

NIÑO

Key words: HUMERAL FRACTURES

ELBOW JOINT

CHILD

\footnotetext{
* Médico Residente de Ortopedia y Traumatología.

† Prof. Adj. de la Clínica de Traumatología y Ortopedia Pediátrica.

‡ Profesor de la Clínica de Traumatología y Ortopedia Pediátrica.

Correspondencia: Dr. Rodrigo Matías Fratelli Álvarez. Correo electrónico: rodrigofratelli@gmail.com

Recibido: $4 / 10 / 17$

Aceptado: 20/1/18
} 


\section{Introducción}

La fractura de epicóndilo medial representa entre $12 \%$ a $20 \%$ de las fracturas del codo pediátrico. La edad más frecuente de presentación es entre los 9 y los 14 años. Se da por una fuerza en valgo en el codo, generalmente en una caída con el brazo y mano extendidos. Existe consenso en la literatura de tratar de forma conservadora a las fracturas no desplazadas (menos de $2 \mathrm{~mm}$ de desplazamiento) y de forma quirúrgica a aquellas fracturas expuestas o con fragmento encarcelado. Existen indicaciones relativas de tratamiento quirúrgico que son la lesión del nervio cubital, una inestabilidad grosera del codo y que se trate de atletas de alto rendimiento ${ }^{(1,2)}$.

La literatura no es clara en la indicación del tratamiento apropiado para las fracturas con un desplazamiento mayor de $2 \mathrm{~mm}$. Existe evidencia a favor de tratar de forma conservadora este tipo de fracturas con buenos resultados funcionales en el seguimiento. Al mismo tiempo hay autores que defienden el tratamiento quirúrgico en estos casos, sobre todo en atletas, para prevenir la inestabilidad en valgo del codo causada por la no unión de estas fracturas que se ha informado hasta en un $60 \%{ }^{(3)}$.

\section{Objetivos del trabajo}

\section{Objetivo general}

El objetivo de este trabajo es revisar la indicación y los resultados de los diferentes tratamientos realizados en las fracturas de epicóndilo medial desplazadas más de 2 $\mathrm{mm}$, sabiendo que hoy día existe acuerdo sobre las indicaciones de tratamiento conservador y quirúrgico con indicaciones establecidas en la literatura revisada. Existe controversia sobre cuál es el mejor tratamiento en las fracturas cerradas, aisladas y desplazadas del epicóndilo medial.

\section{Objetivos especificos}

El objetivo fue comparar:

- Los diferentes estudios de imagen disponibles para el diagnóstico del desplazamiento de la fractura.

- El tratamiento conservador versus el tratamiento quirúrgico en las fracturas desplazadas de epicóndilo medial.

- Complicaciones observadas con los diferentes métodos de tratamiento.

\section{Material y método}

Fuentes de datos: utilizamos las bases de datos electrónicas PubMed y Bireme. La búsqueda fue realizada con las siguientes palabras: Pediatrcic elbow dislocation y Medial epicondyle fracture. Se utilizó el operador boorleano OR entre estos términos. La búsqueda incluyó es-

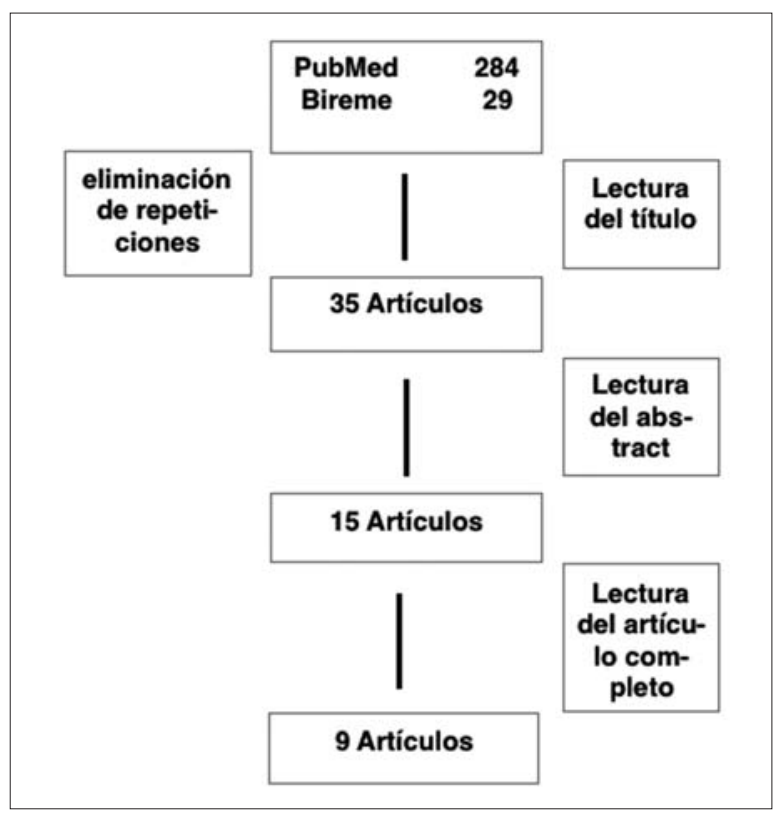

Figura 1. Diagrama de búsqueda

tudios retrospectivos, prospectivos y estudios clínicos publicados en los últimos diez años (agosto de 2006 a agosto de 2016) en los idiomas español e inglés, en humanos y menores de 18 años.

Criterios de inclusión: edad inferior a los 18 años, fracturas de epicóndilo medial desplazadas, estudios que hablen del diagnóstico y resultados del tratamiento de las fracturas desplazadas, estudios que comparen el tratamiento conservador versus el tratamiento quirúrgico.

Criterios de exclusión: estudios que traten de pacientes mayores de 18 años, artículos que hablen solo de fracturas encarceladas o luxo-fracturas de codo, estudios que analicen solo fracturas no desplazadas.

\section{Estrategia de búsqueda}

Se realizó la búsqueda con las palabras claves antes mencionadas, colocando filtros de especie humana, edad, idioma, fecha de publicación y tipos de estudios. Sumando las tres bases de datos se encontraron 313 estudios. Una vez leído el título teniendo en cuenta los criterios de inclusión y exclusión se obtuvieron 35 artículos. Luego de excluir las repeticiones exactas y leer los abstract de los artículos elegidos se redujo a 15 la selección. Por último, al leer el trabajo completo y aplicando los criterios de selección nos quedamos con nueve estudios para analizar (figura 1).

\section{Análisis de artículos}

Se seleccionaron nueve artículos para ser analizados, de los cuales uno es nivel de evidencia I y los restantes 
Tabla 1. Artículos seleccionados.

\begin{tabular}{|c|c|c|c|c|c|c|}
\hline Autor & Tipo de estudio & Nivel de evidencia & $\begin{array}{l}\text { Año y lugar de } \\
\text { publicación }\end{array}$ & $N$ de pacientes & Follow-up (promedio) & Tipo de tratamiento \\
\hline Pace & $\begin{array}{l}\text { Serie de casos. } \\
\text { Retrospectivo }\end{array}$ & IV & $\begin{array}{l}\mathrm{J} \text { of Pediatric Orthop, } \\
2016\end{array}$ & 17 & 11,5 meses & Quirúrgico \\
\hline Lim & $\begin{array}{l}\text { Serie de casos. } \\
\text { Retrospectivo }\end{array}$ & IV & $\begin{array}{l}\mathrm{J} \text { of Pediatric Orthop, } \\
2015\end{array}$ & 34 & 1,4 meses & Conservador \\
\hline Biggers & $\begin{array}{l}\text { Serie de casos. } \\
\text { Retrospectivo }\end{array}$ & IV & $\begin{array}{l}\text { J Surg Orthop Adv, } \\
2015\end{array}$ & 30 & 24 meses & $\begin{array}{l}\text { Conservadorl } \\
\text { Quirúrgico }\end{array}$ \\
\hline Lawrence & $\begin{array}{l}\text { Serie de casos. } \\
\text { Retrospectivo }\end{array}$ & IV & $\begin{array}{c}\text { The American J of } \\
\text { Sports Medicine, } \\
2013\end{array}$ & 20 & 24 meses & $\begin{array}{l}\text { Conservador/ } \\
\text { Quirúrgico }\end{array}$ \\
\hline Park & $\begin{array}{l}\text { Serie de casos. } \\
\text { Comparativo }\end{array}$ & IV & $\begin{array}{c}\text { Yonsei Medical } \\
\text { Journal, } 2012\end{array}$ & 32 & 31 meses & Quirúrgico \\
\hline Shukla & $\begin{array}{l}\text { Serie de casos. } \\
\text { Retrospectivo }\end{array}$ & IV & $\begin{array}{c}\text { Journal of Shoulder } \\
\text { and Elbow Surg, } \\
2011\end{array}$ & 5 & 31 meses & Quirúrgico \\
\hline Edmons & Diagnóstico & I & $\begin{array}{l}\text { The } J \text { of Bone and } \\
\text { Joint Surg, } 2010\end{array}$ & 11 & 12 meses & $\begin{array}{l}\text { Conservadorl } \\
\text { Quirúrgico }\end{array}$ \\
\hline Louahem & $\begin{array}{l}\text { Serie de casos. } \\
\text { Retrospectivo }\end{array}$ & IV & $\begin{array}{c}\text { Archives of } \\
\text { Orthopaedic and } \\
\text { Trauma Surgery, } \\
2010\end{array}$ & 139 & 3,9 años & Quirúrgico \\
\hline Farsetti & Retrospectivo & IV & $\begin{array}{l}\text { The } J \text { of Bone and } \\
\text { Joint Surg, } 2001\end{array}$ & 42 & 45 años & $\begin{array}{l}\text { Conservador/ } \\
\text { Quirúrgico }\end{array}$ \\
\hline
\end{tabular}

Fragmento epicondíleo fuera de la articulación. Lim, Farsetti y Lawrence utilizan como punto de corte $2 \mathrm{~mm}$ para hablar de fractura desplazada al igual que la mayoría de los autores que escriben acerca del tema ${ }^{(1,2)}$. Edmons en su serie define una fractura con desplazamiento mínimo a aquella que presenta menos de $5 \mathrm{~mm}$ de desplazamiento.

ocho nivel IV (tabla 1). El total de pacientes fue 329, se analizaron 331 fracturas de epicóndilo medial (dos pacientes incluidos presentaban fracturas en ambos codos). Con mayoría de varones sobre las mujeres con una relación niño/niña de 1,55/1.

El promedio de edad encontrado en la serie analizada fue de 12,5 años. Del total de fracturas analizadas, 253 fueron tratadas de forma quirúrgica con diferentes técnicas que luego analizaremos, y 78 fueron tratadas de forma conservadora. En la tabla 1 se presentan los artículos con el autor principal, tipo de estudio, nivel de evidencia, lugar y año de publicación, número de pacientes, tiempo de seguimiento promedio y tipo de tratamiento realizado. En las series de Lim, Lawrence, Farsetti y Park se excluyeron los pacientes que presentaran una fractura con el epicóndilo medial con fragmento encarcelado dentro de la articulación. Biggers $(\mathrm{n}=30)$ incluye en su serie cuatro fracturas de este tipo sin dejar claro en su trabajo qué punto de corte toma para llamar desplazada a una fractura. Pace en su serie de 16 pacientes pre- senta dos pacientes con fragmento del epicóndilo encarcelado. En el estudio donde se presenta la serie más extensa de pacientes (Louahem, 2010), de las 139 fracturas analizadas, 68 presentaban una fractura encarcelada en la articulación. Cabe destacar que en este trabajo se incluyen cuatro pacientes que presentaban una lesión muy poco frecuente: una luxación de codo irreductible por tener parte de los músculos epicondíleos interpuestos en el codo con el fragmento epicondíleo fuera de la articulación. Lim, Farsetti y Lawrence utilizan como punto de corte $2 \mathrm{~mm}$ para hablar de fractura desplazada al igual que la mayoría de los autores que escriben sobre el tema ${ }^{(1,2)}$. Edmons en su serie define una fractura con desplazamiento mínimo a aquella que presenta menos de $5 \mathrm{~mm}$ de desplazamiento.

Como variables analizadas y teniendo en cuenta los objetivos específicos se analizaron los métodos diagnósticos utilizados, sobre todo para definir el desplazamiento de la fractura, el tipo de tratamiento realizado, conservador versus quirúrgico, y dentro de los trata- 


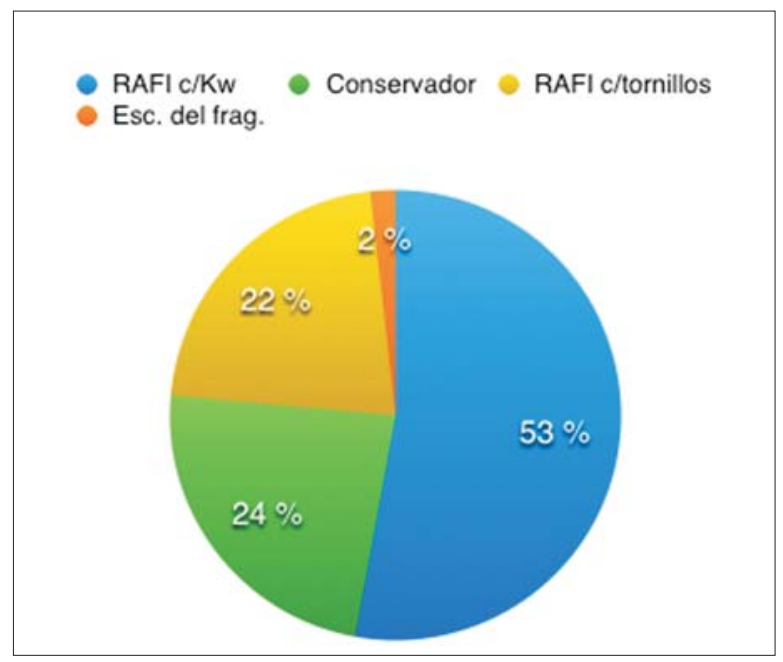

Figura 2. Tipos de tratamientos. RAFI $c / K w n=175$, RAFI c/tornillos $n=72$, escisión del fragmento $n=6$, conservador $\mathrm{n}=78$.

mientos quirúrgicos se analizaron los resultados de las diferentes técnicas utilizadas por los autores en sus trabajos.

\section{Discusión}

\section{Diagnóstico del desplazamiento}

En todos los artículos analizados se utilizaron las radiografías de frente y perfil de codo para llegar al diagnóstico de la fractura de epicóndilo medial. En cuatro de los artículos $^{(6,8,11,12)}$ se realizaron radiografías comparativas con el codo sano, que en algunos de los estudios eran usadas al final del seguimiento para comparar los resultados radiológicos.

En la serie de Biggers se realizaron, una vez diagnosticada la fractura, radiografías en estrés en valgo a todos los pacientes. Se realizaban con 15 grados de flexión y se aplicaba una fuerza en estrés en valgo. Se consideraba positivo si aumentaba más de $1 \mathrm{~mm}$ el sector medial de la interlínea articular comparando con el lado sano.

En la serie de Shukla y de Lawrence se realizaron también radiografías en estrés, sin mencionar cómo las tomaban.

En el artículo de Lim el objetivo fue analizar la tendencia del desplazamiento de la fractura de epicóndilo medial en las diferentes etapas del tratamiento ortopédico con radiografías de frente realizadas al momento de la lesión, a los 20 y 42 días en promedio. Se observó en el $76,5 \%$ de los pacientes la reducción del desplazamiento. Se plantea que esta reducción se deba a la reabsorción del hematoma fracturario y la reducción del edema de las partes blandas que se da sobre todo en la primera semana del tratamiento. Luego de la primera semana los cambios en el desplazamiento no fueron significativos. Para Lim y colaboradores el desplazamiento inicial de la fractura no es el verdadero, sino una sobreestimación falsa debido al hematoma fracturario, concluyendo que el desplazamiento de la fractura de epicóndilo medial se reduce con el tiempo bajo tratamiento ortopédico y esta no es una buena indicación para la cirugía.

Edmonds y colaboradores ${ }^{(11)}$ compararon la exactitud en el diagnóstico del desplazamiento de las radiografías vesus la tomografía computada (TC). Incluyeron fracturas con menos de $5 \mathrm{~mm}$ de desplazamiento a las cuales se les hizo radiografías de frente, perfil, oblicuas interna y externa, y además una TC. En esta serie se sostiene que las radiografías de frente y perfil del codo subestiman el desplazamiento anterior del fragmento así como también el desplazamiento medial. Las radiografías oblicuas internas pueden ser un método seguro para determinar el desplazamiento anterior del fragmento, pero la TC sería el único método exacto para evaluar este desplazamiento.

\section{Tratamiento conservador vesus tratamiento quirúrgico}

Del total de las 331 fracturas analizadas en esta revisión, 253 recibieron tratamiento quirúrgico y 78 tratamiento conservador. La técnica quirúrgica más frecuente observada fue la fijación interna con alambres de Kirschner (Kw) en 174 casos, seguida de la fijación interna con tornillos en 72 pacientes. En los restantes seis pacientes se realizó la escisión del fragmento (figura 2).

En la serie de Park se compararon la fijación con Kw vesus tornillos canulados, tuvieron menor tiempo quirúrgico con la fijación con $\mathrm{Kw}$ (65 minutos promedio vesus 100 minutos con los tornillos). Los tiempos de inmovilización fueron de seis semanas con $\mathrm{Kw}$ y cuatro con tornillos. No hubo diferencias significativas en el score funcional ni en la estabilidad del codo al final del seguimiento.

En el artículo de Louahem de 139 fracturas de epicóndilo medial, 129 fueron tratadas con $\mathrm{Kw}$ y 10 con tornillos canulados; agregaron en la técnica quirúrgica la reparación del cápsulo-ligamentaria medial del codo con sutura en todos los casos. Obtuvieron resultados excelentes en 130 y 9 buenos, sin casos de inestabilidad luego de la cirugía. Cabe destacar que 80 de estos casos fueron luxo-fracturas. A los pacientes que no se presentaron con luxación se les realizó el test en valgo del codo previo a la cirugía siendo positivo en todos casos. Concluye el autor que es más importante la estabilidad en valgo del codo para decidirse por un tratamiento quirúrgico que el desplazamiento inicial de la fractura.

En la serie de Farsetti y colaboradores de 42 fracturas con un follow-up promedio de 45 años se compararon tres tipos de tratamiento: inmovilización con yeso 
Tabla 2. Datos demográficos de los pacientes.

\begin{tabular}{lccc}
\hline Autor & $N$ de pacientes & Edad prom. (años) & M/F \\
\hline Pace & 17 & 13,4 & $9 / 7$ \\
Lim & 34 & 11 & $20 / 14$ \\
Biggers & 30 & 12 & $25 / 5$ \\
Lawrence & 20 & 12,2 & $13 / 7$ \\
Park & 32 & 11,7 & $17 / 14$ \\
Shukla & 5 & 15,8 & $0 / 5$ \\
Edmons & 11 & 12,2 & $4 / 7$ \\
Louahem & 139 & 11,9 & $103 / 67^{*}$ \\
Farsetti & 42 & 12 & $27 / 15$ \\
\hline
\end{tabular}

* Estudio de Louahem diferencia entre M/F de la muestra total $(\mathrm{n}=170)$ y no aclara sexo luego de excluir los pacientes con fracturas no desplazadas. M: masculino; F: femenino.

(19), reducción anatómica y fijación interna con $\mathrm{Kw}$ (17) y escisión del fragmento del epicóndilo medial (6); 17 de los 19 tratados de forma conservadora presentaron pseudoartrosis (PSA) del fragmento del epicóndilo medial, a pesar de esto en los scores funcionales en el seguimiento, 16 tuvieron resultados buenos y 3 moderados. Argumentado que la PSA del epicóndilo medial es una consecuencia asintomática y no una complicación del tratamiento conservador. De los tratados con $\mathrm{Kw}$ todas las fracturas consolidaron con resultados buenos en $15 \mathrm{y}$ dos moderados. En el grupo de la escisión del fragmento se observaron complicaciones como dolor, inestabilidad e hipoplasia del sector medial del húmero distal, con cuatro malos resultados y dos moderados. Los autores concluyen que debe evitarse siempre que sea posible la escisión del fragmento.

En el trabajo de Lawrence de 20 fracturas en adolescentes atletas se compararon dos grupos de pacientes: uno con fracturas de baja energía sin inestabilidad tratados de forma conservadora y otro de fracturas con mayor energía traumática o inestabilidad en valgo en el examen inicial donde se realizó RAFI con tornillos canulados. Los seis del grupo conservador se trataron con un yeso braquipalmar con flexión de $70^{\circ}-90^{\circ}$ y antebrazo neutro por tres a cuatro semanas seguido de una férula posterior que se sacaba para realizar ejercicios hasta las seis semanas, donde se comenzó con fisioterapia. El grupo operado usó una férula por tres semanas y luego siguió el mismo protocolo de rehabilitación. Todos los pacientes lograron la consolidación, con 100\% de satisfacción en el grupo conservador y $93 \%$ en el grupo quirúrgico. Similares resultados funcionales con re- torno de todos los pacientes a sus actividades previas. Los autores enfatizan en que no es el desplazamiento inicial de la fractura el que debe guiar el tratamiento, sino la energía del traumatismo y la estabilidad en valgo del codo. Estas deben ser documentadas y son de gran ayuda para la toma de decisiones.

El estudio de Pace y colaboradores tuvo como objetivo comparar el uso del tornillo con arandela versus el tornillo sin arandela en relación con la fragmentación del epicóndilo al momento de la osteosíntesis, y al mismo tiempo si existía la necesidad de retiro de implante por molestias debido a la arandela. Se realizaron dos grupos de pacientes donde se logró el 100\% de consolidación. Se encontró que el tornillo con arandela necesitó ser removido por molestias en $58 \%$ versus $0 \%$ en el grupo de tornillo único. No hubo casos de fragmentación o penetración del tornillo al momento de la fijación con el uso del tornillo único. Por tanto, concluyen que para el tratamiento de las fracturas de epicóndilo medial el uso de una arandela aumenta la probabilidad de una cirugía adicional debido a molestias del implante en comparación con el uso de tornillo único.

\section{Complicaciones}

Las complicaciones más reportadas en la literatura de las fracturas de epicóndilo medial son la inestabilidad en valgo, la PSA y el compromiso neurológico, sobre todo del nervio cubital. Las dos primeras, inestabilidad y PSA, informadas tras el tratamiento conservador han sido el argumento de varios autores para proponer el tratamiento quirúrgico en las fracturas desplazadas. En los artículos analizados no hubo diferencias significati- 
vas entre el tratamiento conservador y el quirúrgico en cuanto a la inestabilidad en valgo luego del tratamiento. Park y Louahem en sus series de tratamiento con RAFI no tuvieron pacientes con inestabilidad. Farsetti en su trabajo informó cuatro de seis codos inestables luego de la escición del fragmento del epicóndilo con dolor, mostrando resultados malos con este tipo de tratamiento. Biggers en su serie comparativa de 30 pacientes bajo tratamiento conservador versus quirúrgico encontró cuatro pacientes con inestabilidad en valgo; los cuatro habían sido luxo-fracturas, dos tratados de forma quirúrgica y dos ortopédicamente. A pesar de esto tuvieron buenos a excelentes resultados con ambos tratamientos.

Las deformidades anatómicas fueron informadas en casi todos los trabajos, siendo estas relativamente frecuentes en las radiografías, pero no tuvieron implicancias clínicas. Se hallaron hiperplasia e hipoplasia del sector medial del húmero distal, calcificaciones y sulcus en el epicóndilo medial. La frecuencia de estas fue de $16 \%$ en el trabajo de Park, $20 \%$ en el de Louahem, $19 \%$ en el de Farsetti y 19\% en el de Biggers. Estas no tienen efecto sobre la función del codo al final del seguimiento.

En cuanto a las complicaciones neurológicas, todas fueron neuroapraxias en su mayoría del nervio cubital. En la serie de Louahem se informó de un caso de atrapamiento del nervio mediano en la articulación luego de una luxofractura de codo. Cuando el nervio mediano se encuentra afectado hay que sospechar que esté atrapado y estos autores recomiendan realizar cirugía de urgencia para liberarlo. Todos los síntomas neurológicos se resolvieron en un tiempo de tres a seis semanas.

La PSA se ha propuesto como una complicación del tratamiento conservador en fracturas desplazadas de epicóndilo medial. Josefsson y Danielsson ${ }^{(3)}$, en su serie con 35 años de seguimiento, informaban 55\% de PSA con tratamiento conservador. Farsetti $(n=42,2001)$ en su serie con un seguimiento de 45 años promedio encontró $40 \%$ de PSA en el total de pacientes, de los 19 pacientes con fracturas de epicóndilo medial desplazadas tratados con tratamiento conservador, 17 no consolidaron. Sin embargo, 16 de estos obtuvieron buenos resultados funcionales y no hubo inestabilidad al final del seguimiento. No pudiendo demostrar así que el tratamiento conservador genere inestabilidad o limitaciones funcionales al final del seguimiento. En las series analizadas se encontraron dos pacientes con PSA luego de tratamiento quirúrgico. Park informó un paciente con PSA luego de tratamiento con RAFI con tornillo canulado que presentó limitación leve en el rango de movimiento y dificultad en el lanzamiento. El otro caso fue en la serie de Biggers en un paciente que presentó una fractura encarcelada conminuta que requirió RAFI con tornillo más arandela,
Kw y sutura. Este paciente presentó inestabilidad en valgo en el posoperatorio.

\section{Conclusión}

Existen claras indicaciones de tratamiento quirúrgico de las fracturas de epicóndilo medial que ya fueron comentadas. Actualmente no existe consenso en el método apropiado de tratamiento de las fracturas con desplazamiento mayor de $2 \mathrm{~mm}$. De acuerdo a la bibliografía analizada, no debe ser el desplazamiento inicial de la fractura diagnosticado por radiografía lo que decida el tipo de tratamiento. Las radiografías AP y lateral de codo no son suficientes para medir el desplazamiento, las radiografías OI se aproximan más al desplazamiento; sin embargo, el método diagnóstico más preciso para evaluar el desplazamiento es la TC con reconstrucción 3D, como se mostró en el estudio de Edmonds. Por tanto, no solo debemos tener en cuenta el desplazamiento medido por Rx a la hora de tomar decisiones, sino también tener en cuenta la energía traumática y la estabilidad del codo. Conocer bien el mecanismo de la lesión y su energía, sumado a una cuidadosa evaluación de la estabilidad del codo es de suma importancia para la toma de decisiones. Faltan estudios prospectivos para aclarar las ventajas y desventajas del tratamiento conservador versus el quirúrgico. Los datos analizadas en la bibliografía muestran buenos a excelentes resultados con ambos tipos de tratamiento en las fracturas desplazadas del epicóndilo medial. Faltan estudios comparativos con seguimiento a largo plazo, de buen nivel de evidencia, para recomendar uno $\mathrm{u}$ otro tratamiento en las fracturas desplazadas del epicóndilo medial.

\section{Abstract}

Introduction: fractures of medial epicondyle represent 12 to $20 \%$ of pediatric elbow fractures. Treating fractures with greater than $2 \mathrm{~mm}$ of displacement is a matter of debate. The study aims to review indications and results of the different treatments indicated for fractures of medial epicondyle with greater than $2 \mathrm{~mm}$ of displacement.

Method: the PubMed and Bireme electronic data bases were used. Search revealed 313 articles, 9 of which met the inclusion criteria set upon initiation of the study.

Discussion: imaging techniques used and their diagnostic yield were compared, as well as the different of treatments and complications.

Conclusion: treatment should not be defined based on the fracture's displacement, but rather on the trauma energy and the valgus stability of the elbow. Excellent results were reported both with a conservative treatment and with a surgical approach to displaced fractures of 
medial epicondyle. Comparative studies need to be done with long term follow-up that is well recorded to recommend the right treatment for displaced fractures of medial epicondyle.

\section{Resumo}

Introdução: a fratura de epicôndilo medial representa 12 a $20 \%$ das fraturas do cotovelo em crianças. Existe controvérsia na indicação do tratamento para fraturas com deslocamento superior a $2 \mathrm{~mm}$. O objetivo deste trabalho é revisar a indicação e os resultados dos diferentes tratamentos realizados nos casos de fraturas de epicôndilo medial com deslocamento de mais de $2 \mathrm{~mm}$.

Materiais e métodos: foram utilizadas as bases de dados electrónicas PubMed e de Bireme. A pesquisa bibliográfica recuperou 313 artículos sendo que 9 cumpriam com os critérios de inclusão propostos no inicio do trabalho.

Discussão: foram analisados e comparados os métodos de diagnóstico por imagem utilizada e seu rendimento, os diferentes tipos de tratamento $\mathrm{e}$ as complicações.

Conclusão: o deslocamento da fratura não deve ser o determinante do tratamento, mas sim a energia traumática e a estabilidade em valgo do cotovelo. Foram informados resultados excelentes tanto com tratamento conservador como cirúrgico das fraturas com deslocamento de epicôndilo medial. Faltam estudos comparativos com seguimento em longo prazo com evidencias de alta qualidade para recomendar o tipo de tratamento nos casos de fraturas com deslocamento de epicôndilo medial.

\section{Bibliografía}

1. Morrey B. The Elbow and Its Disorders. 3rd.ed. Philadelphia (PA): Saunders, 2004.

2. Skaggs DL, Kocher MS. Master Techniques in Orthopaedic Surgery: Pediatrics. 2nd.ed. Philadelphia (PA): Wolters Kluwer, 2016.
3. Josefsson PO, Danielsson LG. Epicondylar elbow fracture in children. 35-year follow-up of 56 unreduced cases. Acta Orthop Scand 1986; 57:313-5.

4. Lim KB, Woo CY, Chong XL, Ul-Alam S, Allen JC. The isolated medial humeral epicondyle fracture treated nonoperatively: does fracture displacement change over time? J Pediatr Orthop B 2015; 24(3):184-90.

5. Lawrence JT, Patel NM, Macknin J, Flynn JM, Cameron D, Wolfgruber HC, Ganley TJ. Return to competitive sports after medial epicondyle fractures in adolescent athletes: results of operative and nonoperative treatment. Am J Sports Med 2013; 41(5):1152-7.

6. Farsetti P, Potenza V, Caterini R, Ippolito E. Long-term results of treatment of fractures of the medial humeral epicondyle in children. J Bone Joint Surg Am 2001; 83(9):1299-305.

7. Park KB, Kwak YH. Treatment of medial epicondyle fracture without associated elbow dislocation in older children and adolescents. Yonsei Med J 2012; 53(6):1190-6.

8. Biggers M, Bert T, Moisan A. Fracture of the medial humeral epicondyle in children: A comparison of operative or non operative management. J Surg Orthop Adv 2015; 24(3):188-92.

9. Pace GI, Hennrikus WL. Fixation of displaced medial epicondyle fractures in adolescents. J Pediatr Orthop 2016; 37(2):e80-e82

10. Louahem DM, Bourelle S, Buscayret F, Mazeau P, Kelly P, Dimeglio A, et al. Displaced medial epicondyle fractures of the humerus: surgical treatment and results. A report of 139 cases. Arch Orthop Trauma Surg 2010; 130(5):649-55.

11. Edmonds EW. How displaced are "nondisplaced" fractures of the medial humeral epicondyle in children? Results of a three-dimensional computed tomography analysis. J Bone Joint Surg Am 2010; 92(17):2785-91.

12. Shukla SK, Cohen MS. Symptomatic medial epicondyle nonunion: treatment by open reduction and fixation with a tension band construct. J Shoulder Elbow Surg 2011; 20(3):455-60. 